PROCEEDINGS OF THE

AMERICAN MATHEMATICAL SOCIETY

Volume 139, Number 8, August 2011, Pages 2827-2837

S 0002-9939(2011)10762-7

Article electronically published on January 6, 2011

\title{
RANDOM DATA CAUCHY THEORY FOR THE INCOMPRESSIBLE THREE DIMENSIONAL NAVIER-STOKES EQUATIONS
}

\author{
TING ZHANG AND DAOYUAN FANG
}

(Communicated by Matthew J. Gursky)

\begin{abstract}
We study the existence and uniqueness of the strong solution for the incompressible Navier-Stokes equations with the $L^{2}$ initial data and the periodic space domain $\mathbb{T}^{3}$. After a suitable randomization, we are able to construct the local unique strong solution for a large set of initial data in $L^{2}$. Furthermore, if $\left\|u_{0}\right\|_{L^{2}}$ is small, we show that the probability for the global existence and uniqueness of the solution is large.
\end{abstract}

\section{INTRODUCTION}

In this paper, we are going to study the incompressible periodic Navier-Stokes system, namely,

$$
\left\{\begin{array}{l}
u_{t}+u \cdot \nabla u-\Delta u+\nabla \Pi=0, x \in \mathbb{T}^{3}, t>0, \\
\operatorname{div} u=0, \\
\left.u\right|_{t=0}=u_{0},
\end{array}\right.
$$

where $u(t, x)$ and $\Pi(t, x)$ denote the fluid velocity and the pressure, respectively, the three dimensional torus $\mathbb{T}^{3}:=(\mathbb{R} / 2 \pi \mathbb{Z})^{3}$.

The existence of at least one weak solution of (1.1) has been well-known since the pioneering work of Leray [13] and Hopf [8. To prove the uniqueness of the solution in some time interval $[0, T)$, we need besides the condition $u_{0} \in L^{2}$ a further regularity property of the initial value $u_{0}$. The first sufficient condition on the initial value for a bounded domain seems to have been described in [11. Then many results on sufficient conditions on $u_{0}$ to guarantee the existence and uniqueness of the solution were proved; see, e.g., 1, 4, 6, 7, 9, 12, In [5, Farwig and Sohr gave the weakest necessary and sufficient condition when the space is a bounded domain with $C^{2,1}$ boundary. These well-posedness theories are valid all the way down to the scaling regularity. In [3], Burq and Tzvetkov considered the local well-posedness theory for the cubic nonlinear wave equation with the initial data in the supercritical space. Using Burq and Tzvetkov's method, we will obtain

Received by the editors December 2, 2009 and, in revised form, March 10, 2010 and July 27, 2010 .

2010 Mathematics Subject Classification. Primary 35Q30; Secondary 76D05, 35A01, 35A02.

Key words and phrases. Navier-Stokes equations, existence and uniqueness.

The authors were supported in part by the National Natural Science Foundation of China (NSFC) $(10871175,10931007,10901137)$, the Zhejiang Provincial Natural Science Foundation of China (Z6100217), and SRFDP No. 20090101120005.

(C)2011 American Mathematical Society Reverts to public domain 28 years from publication 
the existence and uniqueness of the local solution in "a large class of initial data in $L_{\sigma}^{2}\left(\mathbb{T}^{3}\right) "$.

Let us first explain what we mean by "a large class of initial data in $L_{\sigma}^{2}\left(\mathbb{T}^{3}\right)$ ", where $L_{\sigma}^{2}\left(\mathbb{T}^{3}\right)=\overline{C_{\sigma}^{\infty}\left(\mathbb{T}^{3}\right)}\|\cdot\|_{L^{2}}, C_{\sigma}^{\infty}\left(\mathbb{T}^{3}\right)=\left\{u \in C^{\infty}\left(\mathbb{T}^{3}\right): \operatorname{div} u=0, \int_{\mathbb{T}^{3}} u d x=0\right\}$. Define $\mathbb{Z}_{+}^{3}=\left\{k \in \mathbb{Z}^{3} ; k_{1}>0\right\} \cup\left\{k \in \mathbb{Z}^{3} ; k_{1}=0, k_{2}>0\right\} \cup\left\{k \in \mathbb{Z}^{3} ; k_{1}=0, k_{2}=\right.$ $\left.0, k_{3}>0\right\}, \mathbb{Z}_{-}^{3}=-\mathbb{Z}_{+}^{3}$ and $\mathbb{Z}_{*}^{3}=\mathbb{Z}_{+}^{3} \cup \mathbb{Z}_{-}^{3}$. Let $k^{\perp}=\left\{\eta \in \mathbb{R}^{3} ; k \cdot \eta=0\right\},\left\{e_{k, 1}, e_{k, 2}\right\}$ be an orthonormal basis of $k^{\perp}$. Let $e_{k}(x)=\cos (k \cdot x)$ if $k \in \mathbb{Z}_{+}^{3}, e_{k}(x)=\sin (k \cdot x)$ if $k \in \mathbb{Z}_{-}^{3}$ denote

$$
e_{k}^{i}(x)=e_{k}(x) e_{k, i}, \forall k \in \mathbb{Z}_{*}^{3}, i=1,2 .
$$

Then, $\left\{e_{k}^{i} ; k \in \mathbb{Z}_{*}^{3}, i=1,2\right\}$ is a Fourier basis of $L_{\sigma}^{2}\left(\mathbb{T}^{3}\right)$ (up to the constant $\frac{\sqrt{2}}{(2 \pi)^{\frac{3}{2}}}$ ). Let $\left\{h_{k}^{i}(\omega)\right\}_{k \in \mathbb{Z}_{*}^{3}, i=1,2}$ be a sequence of independent, 0 mean value, real random variables on a probability space $(\Omega, \mathcal{F}, P)$ such that

$$
\exists C>0, \forall k \in \mathbb{Z}_{*}^{3}, i=1,2, \int_{\Omega}\left|h_{k}^{i}(\omega)\right|^{4} d P(\omega) \leq C .
$$

For $u_{0} \in L_{\sigma}^{2}\left(\mathbb{T}^{3}\right)$, i.e.,

$$
u_{0}(x)=\sum_{k \in \mathbb{Z}_{*}^{3}} \sum_{i=1}^{2} \alpha_{k}^{i} e_{k}^{i}, \text { where } \sum_{k \in \mathbb{Z}_{*}^{3}} \sum_{i=1}^{2}\left(\alpha_{k}^{i}\right)^{2}<\infty,
$$

we consider the map

$$
\omega \mapsto u_{0}^{\omega}
$$

from probability space $(\Omega, \mathcal{F})$ to $L_{\sigma}^{2}\left(\mathbb{T}^{3}\right)$ equipped with Borel sigma algebra, defined by

$$
u_{0}^{\omega}(x)=\sum_{k \in \mathbb{Z}_{*}^{3}} \sum_{i=1}^{2} h_{k}^{i}(\omega) \alpha_{k}^{i} e_{k}^{i}
$$

One can check that the map $\omega \mapsto u_{0}^{\omega}$ is measurable and $u_{0}^{\omega} \in L^{2}\left(\Omega ; L_{\sigma}^{2}\left(\mathbb{T}^{3}\right)\right)$. Thus it defines a $L_{\sigma}^{2}\left(\mathbb{T}^{3}\right)$ valued random variable. From Appendix 2 in 3 , one can obtain that if $u_{0} \notin H^{\varepsilon}\left(\mathbb{T}^{3}\right), \varepsilon>0$, for almost all $\omega, u_{0}^{\omega}$ does not belongs to $H^{\varepsilon}\left(\mathbb{T}^{3}\right)$; i.e., the randomization $\omega \mapsto u_{0}^{\omega}$ does not give a regularization in the scale of the Sobolev spaces. But, the $L^{p}$ regularization of this randomization will play an important role in this paper. Our main results read as follows.

Theorem 1.1 (Local existence and uniqueness). Let us fix $u_{0} \in L_{\sigma}^{2}\left(\mathbb{T}^{3}\right)$, and let $u_{0}^{\omega}$ be defined by the randomization (1.4)-(1.5). Then for almost all $\omega \in \Omega$, there exist $T_{\omega}^{*}>0$ and a unique solution to (1.1) with the initial data $u_{0}^{\omega}$ in the space

$$
\begin{aligned}
\{u: & t^{\frac{1}{2}-\frac{3}{2 p}} u(u) \in C\left(\left[0, T_{\omega}^{*}\right) ; L^{p}\left(\mathbb{T}^{3}\right)\right), p \in[3, \infty], \\
& \left.t^{1-\frac{3}{2 q}} \nabla u(t) \in C\left(\left[0, T_{\omega}^{*}\right) ; L^{q}\left(\mathbb{T}^{3}\right)\right), q \in[3, \infty)\right\} .
\end{aligned}
$$

In the proof of Theorem 1.1. we use Lemma 2.3 (obtained from Lemmas 3.1 and 4.2 in [3] or the Khinchin inequality [10]) to show that $u_{0}^{\omega} \in L^{3}$ for almost all $\omega \in \Omega$ in Proposition 2.4, and we use Kato's result [9] to get the local existence and uniqueness of the local solution. In the following theorem, we will estimate the probability of the event $\left\{\omega \in \Omega: T_{\omega}^{*}=+\infty\right\}$. 
Theorem 1.2 (Global existence and uniqueness). Under the conditions in Theorem 1.1, there exist $C>0$ and an event $\Omega_{\infty}$ such that

$$
P\left(\Omega_{\infty}\right) \geq 1-C\left\|u_{0}\right\|_{L^{2}}^{3},
$$

and for every $\omega \in \Omega_{\infty}$ there exists a unique global solution $u^{\omega}$ of (1.1) with the initial data $u_{0}^{\omega}$ in the space (1.6) with $T_{\omega}^{*}=+\infty$.

Moreover, if the random variables $h_{k}^{i}$ are standard real Gaussian or Bernoulli variables, then (1.7) can be improved to

$$
P\left(\Omega_{\infty}\right) \geq 1-C e^{-\left(C\left\|u_{0}\right\|_{L^{2}}\right)^{-2}}, C>0 .
$$

Remark 1.3. This theorem means that if $\left\|u_{0}\right\|_{L^{2}}$ is small, the probability for the global existence and uniqueness of the solution is large.

By using Kato's result $\left[9\right.$, we cannot estimate $\left\{\omega \in \Omega: T_{\omega}^{*}>T\right\}$ directly. Thus, we need an additional condition

$$
\exists C>0, \forall k \in \mathbb{Z}_{*}^{3}, i=1,2, \int_{\Omega}\left|h_{k}^{i}(\omega)\right|^{6} d P(\omega) \leq C,
$$

and use the smallness of the time $T$ to control the smallness of the linear solution $\left\|e^{t \Delta} u_{0}^{\omega}\right\|_{L^{6}\left(\Omega ; L^{4}\left([0, T] ; L^{6}\left(\mathbb{T}^{3}\right)\right)\right)}$ and $\left\|\nabla e^{t \Delta} u_{0}^{\omega}\right\|_{L^{6}\left(\Omega ; L^{2}\left([0, T] ; L^{3}\left(\mathbb{T}^{3}\right)\right)\right)}$ in Propositions 3.1 and 3.2 . Finally, we will estimate the probability of the event $\left\{\omega \in \Omega: T_{\omega}^{*}>T\right\}$ in the following theorem.

Theorem 1.4. Under the conditions in Theorem 1.1, if the random variables $h_{k}^{i}$ satisfy (1.9), then for almost all $\omega \in \Omega$ there exist $T_{\omega}>0$ and a unique solution to (1.1) with the initial data $u_{0}^{\omega}$ in a space continuously embedded in

$$
X_{\omega}=e^{t \Delta} u_{0}^{\omega}+C\left(\left[0, T_{\omega}\right] ; H^{\frac{1}{2}}\left(\mathbb{T}^{3}\right)\right) \cap L^{2}\left(\left[0, T_{\omega}\right] ; H^{\frac{3}{2}}\left(\mathbb{T}^{3}\right)\right) .
$$

More precisely, there exist $C>0$, and for every $0<T \leq 1$ an event $\Omega_{T}$ such that

$$
P\left(\Omega_{T}\right) \geq 1-C\left(\left\|u_{0}\right\|_{L^{2}\left(\mathbb{T}^{3}\right)} T^{\frac{1}{4}}+\left\|\alpha_{k}^{i} \sqrt{1-e^{-2|k|^{2} T}}\right\|_{l_{k, i}^{2}}\right)^{6},
$$

and for every $\omega \in \Omega_{T}$ there exists a unique solution $u^{\omega}$ of (1.1) with the initial data $u_{0}^{\omega}$ in a space continuously embedded in $C\left([0, T] ; L^{2}\left(\mathbb{T}^{3}\right)\right) \cap L^{2}\left([0, T] ; H^{1}\left(\mathbb{T}^{3}\right)\right)$.

Moreover, if the random variables $h_{k}^{i}$ are standard real Gaussian or Bernoulli variables, then (1.10) can be improved to

$$
P\left(\Omega_{T}\right) \geq 1-C \exp \left(-\frac{c}{\left\|u_{0}\right\|_{L^{2}\left(\mathbb{T}^{3}\right)}^{2} T^{\frac{1}{2}}+\| \alpha_{k}^{i} \sqrt{1-e^{-2|k|^{2} T} \|_{l_{k, i}^{2}}^{2}}}\right), C, c>0 .
$$

Remark 1.5. Since $u_{0} \in L_{\sigma}^{2}\left(\mathbb{T}^{3}\right)$, it is easy to see that

$$
\lim _{T \rightarrow 0} P\left(\Omega_{T}\right)=1 .
$$

Our results show that in some sense the problem (1.1) is local well-posed for almost all initial data in $L_{\sigma}^{2}\left(\mathbb{T}^{3}\right)$, exhibiting a gain of $\frac{1}{2}$ derivatives with respect to the critical Hilbert space $H^{\frac{1}{2}}\left(\mathbb{T}^{3}\right)$. 
Remark 1.6. As that in [3], the method of proof consists in using the fact that although the initial data have low regularity, their $L^{p}$ properties are (almost surely) much better than expected, allowing the use of a fixed point method after having singled out the linear evolution. Such $L^{p}$ regularization phenomena have been wellknown in the context of Fourier series since the work of Paley-Zygmund [14, and Ayache and Tzvetkov studied similar phenomena in [2].

Remark 1.7. From the estimate of $\left\|e^{t \Delta} u_{0}^{\omega}\right\|_{L_{t}^{4} L_{x}^{6}}$ in Proposition 3.1 and similar arguments as that in [5, one can also obtain that there exist $T_{\omega}>0$ and a unique solution to (1.1) with the initial data $u_{0}^{\omega}$ in a space continuously embedded in $L^{4}\left(\left[0, T_{\omega}\right] ; L^{6}\left(\mathbb{T}^{3}\right)\right)$. Then (1.10) can be improved to

$$
P\left(\Omega_{T}\right) \geq 1-C\left(\left\|u_{0}\right\|_{L^{2}\left(\mathbb{T}^{3}\right)} T^{\frac{1}{4}}\right)^{6},
$$

and (1.11) can be improved to

$$
P\left(\Omega_{T}\right) \geq 1-C \exp \left(-\frac{c}{\left\|u_{0}\right\|_{L^{2}\left(\mathbb{T}^{3}\right)}^{2} T^{\frac{1}{2}}}\right), C, c>0 .
$$

This means that considering the different solution spaces, we would have different estimates of $P\left(\Omega_{T}\right)$.

\section{EXISTEnCE AND UNIQUENESS}

In this section, we prove the existence and uniqueness of the solution for the 3D incompressible Navier-Stokes equations. The following lemmas were proved in [3].

Lemma 2.1 ([3, Lemma 4.2). Assume that $\left\{l_{n}(\omega)\right\}_{n=1}^{\infty}$ is a sequence of independent, 0-mean value, complex random variables satisfying for some $k \in \mathbb{N}^{*}$,

$$
\exists C>0, \forall n \geq 1, \int_{\Omega}\left|l_{n}(\omega)\right|^{2 k} d P(\omega) \leq C .
$$

Then

(2.1) $\forall 2 \leq p \leq 2 k, \exists C>0, \forall\left\{c_{n}\right\}_{n \in \mathbb{N}^{*}} \in l^{2}\left(\mathbb{N}^{*}, \mathbb{C}\right),\left\|\sum_{n} c_{n} l_{n}\right\|_{L^{p}(\Omega)} \leq C\left\|c_{n}\right\|_{l^{2}}$.

Lemma 2.2 ([3], Lemma 3.1). Let $\left\{l_{n}(\omega)\right\}_{n=1}^{\infty}$ be a sequence of independent, 0mean value, real random variables with associated sequence of distributions $\left\{\mu_{n}\right\}_{n=1}^{\infty}$. Assume that $\mu_{n}$ satisfy the property

$$
\exists c>0: \forall \gamma \in \mathbb{R}, \forall n \geq 1,\left|\int_{-\infty}^{\infty} e^{\gamma x} d \mu_{n}(x)\right| \leq e^{c \gamma^{2}} .
$$

Then there exists $\alpha>0$ such that for every $\lambda>0$ and every sequence $\left\{c_{n}\right\}_{n=1}^{\infty} \in l^{2}$ of real numbers,

$$
P\left(\omega:\left|\sum_{n=1}^{\infty} c_{n} l_{n}(\omega)\right|>\lambda\right) \leq 2 e^{-\frac{\alpha \lambda^{2}}{\sum c_{n}^{2}}} .
$$

As a consequence there exists $C>0$ such that for every $p \geq 2$ and every sequence $\left\{c_{n}\right\}_{n=1}^{\infty} \in l^{2}$,

$$
\left\|\sum_{n=1}^{\infty} c_{n} l_{n}(\omega)\right\|_{L^{p}(\Omega)} \leq C \sqrt{p}\left(\sum_{n=1}^{\infty} c_{n}^{2}\right)^{\frac{1}{2}} .
$$


Then, from Lemmas 2.12.2 (or the Khinchin inequality [10]), we can immediately obtain the following lemma.

Lemma 2.3. Assume that $\left\{h_{k}^{i}\right\}_{k \in \mathbb{Z}_{*}^{3}, i=1,2}$ is a sequence of independent, 0-mean value, real random variables satisfying

$$
\exists C>0, \forall k \in \mathbb{Z}_{*}^{3}, i=1,2, \int_{\Omega}\left|h_{k}^{i}(\omega)\right|^{2} k d P(\omega) \leq C,
$$

for some $k \in \mathbb{N}^{*}$. Then there exists $C>0$ such that for every sequence $\left\{c_{k}^{i}\right\}_{k \in \mathbb{Z}_{*}^{3}, i=1,2}$ $\in l_{k, i}^{2}$,

$$
\left\|\sum_{k \in \mathbb{Z}_{*}^{3}} \sum_{i=1}^{2} c_{k}^{i} h_{k}^{i}(\omega)\right\|_{L^{q}(\Omega)} \leq C\left\|c_{k}^{i}\right\|_{l_{k, i}^{2}}, q \in[2,2 k] .
$$

Moreover, if $h_{k}^{i}$ are standard real Gaussian or Bernoulli variables, then there exists $C>0$ such that for every $p \geq 2$ and every sequence $\left\{c_{k}^{i}\right\}_{k \in \mathbb{Z}_{*}^{3}, i=1,2} \in l_{k, i}^{2}$,

$$
\left\|\sum_{k \in \mathbb{Z}_{*}^{3}} \sum_{i=1}^{2} c_{k}^{i} h_{k}^{i}(\omega)\right\|_{L^{p}(\Omega)} \leq C \sqrt{p}\left\|c_{k}^{i}\right\|_{l_{k, i}^{2}} .
$$

Proposition 2.4. Assume that $u_{0} \in L_{\sigma}^{2}\left(\mathbb{T}^{3}\right),\left\{h_{k}^{i}\right\}_{k \in \mathbb{Z}_{*}^{3}, i=1,2}$ is a sequence of independent, 0-mean value, real random variables satisfying (1.2). Then there exists $C>0$ such that for every $u_{0} \in L_{\sigma}^{2}\left(\mathbb{T}^{3}\right)$ we have

$$
\left\|u_{0}^{\omega}\right\|_{L^{3}\left(\Omega ; L^{3}\left(\mathbb{T}^{3}\right)\right)} \leq C\left\|u_{0}\right\|_{L^{2}\left(\mathbb{T}^{3}\right)} .
$$

In particular, if we set

$$
D_{\lambda, u_{0}}=\left\{\omega \in \Omega:\left\|u_{0}^{\omega}\right\|_{L^{3}\left(\mathbb{T}^{3}\right)} \geq \lambda\right\},
$$

from the Bienaymé-Tchebichev inequality we obtain that there exists $C>0$ such that for every $\lambda>0$ and every $u_{0} \in L_{\sigma}^{2}\left(\mathbb{T}^{3}\right)$,

$$
P\left(D_{\lambda, u_{0}}\right) \leq C \lambda^{-3}\left\|u_{0}\right\|_{L^{2}}^{3} .
$$

Proof. Using the Minkowski inequality and Lemma 2.3. we have

$$
\begin{aligned}
\left\|u_{0}^{\omega}\right\|_{L^{3}\left(\Omega ; L^{3}\left(\mathbb{T}^{3}\right)\right)} & =\left\|\sum_{k \in \mathbb{Z}_{*}^{3}} \sum_{i=1}^{2} h_{k}^{i}(\omega) \alpha_{k}^{i} e_{k}^{i}\right\|_{L^{3}\left(\mathbb{T}^{3} ; L^{3}(\Omega)\right)} \\
& \leq C\left\|\left(\sum_{k \in \mathbb{Z}_{*}^{3}} \sum_{i=1}^{2}\left|\alpha_{k}^{i} e_{k}^{i}\right|^{2}\right)^{\frac{1}{2}}\right\|_{L^{3}\left(\mathbb{T}^{3}\right)} \\
& \leq C\left(\sum_{k \in \mathbb{Z}_{*}^{3}} \sum_{i=1}^{2}\left\|\alpha_{k}^{i} e_{k}^{i}\right\|_{L^{3}\left(\mathbb{T}^{3}\right)}^{2}\right)^{\frac{1}{2}} \\
& \leq C\left\|\alpha_{k}^{i}\right\|_{l_{k, i}^{2}}=C\left\|u_{0}\right\|_{L^{2}\left(\mathbb{T}^{3}\right)}
\end{aligned}
$$

Using the Bienaymé-Tchebichev inequality, we obtain

$$
P\left(E_{\lambda, u_{0}}\right) \leq \lambda^{-3}\left(C\left\|u_{0}\right\|_{L^{2}}\right)^{3} .
$$


Theorem 2.5 (T. Kato, 1972, 9]). If $u_{0} \in L^{3}$, then there exists $T^{*}>0$ such that there exists a unique solution to (1.1) with the initial data $u_{0}$ in the space

$$
\begin{aligned}
\{u: & t^{\frac{1}{2}-\frac{3}{2 p}} u(u) \in C\left(\left[0, T_{\omega}^{*}\right) ; L^{p}\left(\mathbb{T}^{3}\right)\right), p \in[3, \infty], \\
& \left.t^{1-\frac{3}{2 q}} \nabla u(t) \in C\left(\left[0, T_{\omega}^{*}\right) ; L^{q}\left(\mathbb{T}^{3}\right)\right), q \in[3, \infty)\right\} .
\end{aligned}
$$

Furthermore, there exists a constant $c_{1}>0$ such that

$$
\left\|u_{0}\right\|_{L^{3}} \leq c_{1} \Rightarrow T^{*}=+\infty .
$$

Proof of Theorem 1.1. From Proposition 2.4, we have that $u_{0}^{\omega} \in L^{3}\left(\mathbb{T}^{3}\right)$ for almost all $\omega \in \Omega$. Then, from Theorem 2.5 one can obtain the local existence and uniqueness of the solution and finish the proof of Theorem 1.1

Proposition 2.6. Under the assumptions of Proposition 3.1, if $h_{k}^{i}$ are standard real Gaussian or Bernoulli variables, then there exists $C>0$ such that for every $u_{0} \in L_{\sigma}^{2}\left(\mathbb{T}^{3}\right), p \geq 3$, we have

$$
\left\|u_{0}^{\omega}\right\|_{L^{p}\left(\Omega ; L^{3}\left(\mathbb{T}^{3}\right)\right)} \leq C \sqrt{p}\left\|u_{0}\right\|_{L^{2}\left(\mathbb{T}^{3}\right)} .
$$

In particular, if we set

$$
\bar{D}_{\lambda, u_{0}}=\left\{\omega \in \Omega:\left\|u_{0}^{\omega}\right\|_{L^{3}\left(\mathbb{T}^{3}\right)} \geq \lambda\right\}
$$

from the Bienaymé-Tchebichev inequality, we obtain that there exist $C>0$ and $c>0$ such that for every $\lambda>0$ and every $u_{0} \in L_{\sigma}^{2}\left(\mathbb{T}^{3}\right)$,

$$
P\left(\bar{D}_{\lambda, u_{0}}\right) \leq C e^{-\left(\frac{c \lambda}{\left\|u_{0}\right\|_{L^{2}}}\right)^{2}} \text {. }
$$

Proof. Using a similar argument as that in the proof of (2.8), we can easily obtain (2.11).

Then, using the Bienaymé-Tchebichev inequality, we obtain that there exists $\alpha>0$ such that for every $p \geq 3$ and every $u_{0} \in L^{2}\left(\mathbb{T}^{3}\right)$,

$$
P\left(\bar{D}_{\lambda, u_{0}}\right) \leq \lambda^{-p}\left(\alpha \sqrt{p}\left\|u_{0}\right\|_{L^{2}}\right)^{p} .
$$

If $\lambda$ satisfies

$$
\frac{\lambda}{\left\|u_{0}\right\|_{L^{2}}} \leq 3 \alpha e
$$

then

$$
C e^{-\left(\frac{c \lambda}{\left\|u_{0}\right\|_{L^{2}}}\right)^{2}} \geq C e^{-(3 c \alpha e)^{2}},
$$

and we can find some $C>0$ and $c>0$ such that

$$
C e^{-(3 c \alpha e)^{2}} \geq 1 \geq P\left(\bar{D}_{\lambda, u_{0}}\right) .
$$

Thus inequality (2.12) holds if $\lambda$ satisfies (2.14). If (2.14) does not hold, we set

$$
p:=\left(\frac{\lambda}{\alpha e\left\|u_{0}\right\|_{L^{2}}}\right)^{2} \geq 9,
$$

and from (2.13) we obtain

$$
P\left(\bar{D}_{\lambda, u_{0}}\right) \leq\left(\frac{\alpha\left\|u_{0}\right\|_{L^{2}}}{\lambda} \cdot \frac{\lambda}{\alpha e\left\|u_{0}\right\|_{L^{2}}}\right)^{\left(\frac{\lambda}{\alpha e\left\|u_{0}\right\|_{L^{2}}}\right)^{2}}=e^{-\left(\frac{\lambda}{\alpha e\left\|u_{0}\right\|_{L^{2}}}\right)^{2}} .
$$


Proof of Theorem 1.2, Let

$$
\Omega_{\infty}=D_{c_{1}, u_{0}}^{c} .
$$

From (2.9) we have (1.7). For any $\omega \in \Omega_{\infty}$, we have

$$
\left\|u_{0}^{\omega}\right\|_{L^{3}\left(\mathbb{T}^{3}\right)}<c_{1} .
$$

From Theorem 2.5 we have $T_{\omega}^{*}=+\infty$.

Moreover, if the random variables $h_{k}^{i}$ are standard real Gaussian or Bernoulli variables, then from (2.12) we can let

$$
\Omega_{\infty}=\bar{D}_{c_{1}, u_{0}}^{c},
$$

obtain (1.8), and finish the proof of Theorem 1.2 .

\section{The estimate of $P\left(\Omega_{T}\right)$}

In this section, we will prove Theorem 1.4. Although the proof of the existence and uniqueness of the local solution is classical, for the convenience of the reader, we also give the proofs. First we estimate the linear solution $e^{t \Delta} u_{0}^{\omega}$.

Proposition 3.1. For $u_{0} \in L_{\sigma}^{2}\left(\mathbb{T}^{3}\right)$, we consider the free evolution with the initial data $u_{0}^{\omega}$ given by

$$
\bar{u}^{\omega}(t, x)=e^{t \Delta} u_{0}^{\omega} .
$$

Assume that $\left\{h_{k}^{i}\right\}_{k \in \mathbb{Z}_{*}^{3}, i=1,2}$ is a sequence of independent, 0 -mean value, real random variables satisfying (1.9). Then, for any $0<T \leq 1$, we have

$$
\begin{gathered}
\left\|\bar{u}^{\omega}\right\|_{L^{6}\left(\Omega ; L^{4}\left([0, T] ; L^{6}\left(\mathbb{T}^{3}\right)\right)\right)} \leq C\left\|u_{0}\right\|_{L^{2}\left(\mathbb{T}^{3}\right)} T^{\frac{1}{4}}, \\
\left\|\nabla \bar{u}^{\omega}\right\|_{L^{6}\left(\Omega ; L^{2}\left([0, T] ; L^{3}\left(\mathbb{T}^{3}\right)\right)\right)} \leq C\left\|\alpha_{k}^{i} \sqrt{1-e^{-2|k|^{2} T}}\right\|_{l_{k, i}^{2}}, \\
P\left(E_{\lambda, T, u_{0}}\right) \leq C \lambda^{-6}\left(\left\|u_{0}\right\|_{L^{2}\left(\mathbb{T}^{3}\right)} T^{\frac{1}{4}}+\left\|\alpha_{k}^{i} \sqrt{1-e^{-2|k|^{2} T}}\right\|_{l_{k, i}^{2}}\right)^{6},
\end{gathered}
$$

where $\lambda>0, C>0$ is independent of $T$, and

$$
E_{\lambda, T, u_{0}}=\left\{\omega \in \Omega:\left\|\bar{u}^{\omega}\right\|_{L^{4}\left([0, T] ; L^{6}\left(\mathbb{T}^{3}\right)\right)}+\left\|\nabla \bar{u}^{\omega}\right\|_{L^{2}\left([0, T] ; L^{3}\left(\mathbb{T}^{3}\right)\right)} \geq \lambda\right\} .
$$

Proof. Using the Minkowski inequality and Lemma 2.3 , we have, for any $T \in(0,1]$,

$$
\begin{aligned}
\left\|\bar{u}^{\omega}\right\|_{L^{6}\left(\Omega ; L^{4}\left([0, T] ; L^{6}\left(\mathbb{T}^{3}\right)\right)\right)} & \leq\left\|\sum_{k \in \mathbb{Z}_{*}^{3}} \sum_{i=1}^{2} h_{k}^{i}(\omega) e^{-|k|^{2} t} \alpha_{k}^{i} e_{k}^{i}\right\|_{L^{4}\left([0, T] ; L^{6}\left(\mathbb{T}^{3} ; L^{6}(\Omega)\right)\right)} \\
& \leq C\left\|\left(\sum_{k \in \mathbb{Z}_{*}^{3}} \sum_{i=1}^{2} e^{-2|k|^{2} t}\left|\alpha_{k}^{i} e_{k}^{i}\right|^{2}\right)^{\frac{1}{2}}\right\|_{L^{4}\left([0, T] ; L^{6}\left(\mathbb{T}^{3}\right)\right)} \\
& \leq C\left(\sum_{k \in \mathbb{Z}_{*}^{3}} \sum_{i=1}^{2}\left\|e^{-|k|^{2} t} \mid \alpha_{k}^{i} e_{k}^{i}\right\|_{L^{4}\left([0, T] ; L^{6}\left(\mathbb{T}^{3}\right)\right)}^{2}\right)^{2} \\
& \leq C T^{\frac{1}{4}}\left\|\alpha_{k}^{i}\right\|_{l_{k, i}^{2}}=C\left\|u_{0}\right\|_{L^{2}\left(\mathbb{T}^{3}\right)} T^{\frac{1}{4}}
\end{aligned}
$$


and

$$
\begin{aligned}
\left\|\nabla \bar{u}^{\omega}\right\|_{L^{6}\left(\Omega ; L^{2}\left([0, T] ; L^{3}\left(\mathbb{T}^{3}\right)\right)\right)} & \leq\left\|\sum_{k \in \mathbb{Z}_{*}^{3}} \sum_{i=1}^{2} h_{k}^{i}(\omega) e^{-|k|^{2} t} k \alpha_{k}^{i} e_{k}^{i}\right\|_{L^{2}\left([0, T] ; L^{3}\left(\mathbb{T}^{3} ; L^{6}(\Omega)\right)\right)} \\
& \leq C\left\|\left(\sum_{k \in \mathbb{Z}_{*}^{3}} \sum_{i=1}^{2} e^{-2|k|^{2} t}|k|^{2}\left|\alpha_{k}^{i} e_{k}^{i}\right|^{2}\right)^{\frac{1}{2}}\right\|_{L^{2}\left([0, T] ; L^{3}\left(\mathbb{T}^{3}\right)\right)} \\
& \leq C\left(\sum _ { k \in \mathbb { Z } _ { * } ^ { 3 } } \sum _ { i = 1 } ^ { 2 } \left\|e^{-|k|^{2} t}\left|k\left\|\alpha_{k}^{i} e_{k}^{i} \mid\right\|_{L^{2}\left([0, T] ; L^{3}\left(\mathbb{T}^{3}\right)\right)}^{2}\right)^{2}\right.\right. \\
& \leq C\left\|\alpha_{k}^{i} \sqrt{1-e^{-2|k|^{2} T}}\right\|_{l_{k, i}^{2}} .
\end{aligned}
$$

Using the Bienaymé-Tchebichev inequality, we can obtain (3.3).

Proposition 3.2. Under the assumptions of Proposition 3.1, if $h_{k}^{i}$ are standard real Gaussian or Bernoulli variables, then there exists $C>0$ such that for every $u_{0} \in L_{\sigma}^{2}\left(\mathbb{T}^{3}\right), p \geq 6,0<T \leq 1$, we have

$$
\begin{gathered}
\left\|\bar{u}^{\omega}\right\|_{L^{p}\left(\Omega ; L^{4}\left([0, T] ; L^{6}\left(\mathbb{T}^{3}\right)\right)\right)} \leq C \sqrt{p}\left\|u_{0}\right\|_{L^{2}\left(\mathbb{T}^{3}\right)} T^{\frac{1}{4}} \\
\left\|\nabla \bar{u}^{\omega}\right\|_{L^{p}\left(\Omega ; L^{2}\left([0, T] ; L^{3}\left(\mathbb{T}^{3}\right)\right)\right)} \leq C \sqrt{p}\left\|\alpha_{k}^{i} \sqrt{1-e^{-2|k|^{2} T}}\right\|_{l_{k, i}^{2}}, \\
P\left(F_{\lambda, T, u_{0}}\right) \leq C \exp \left(-\frac{c \lambda^{2}}{\left\|u_{0}\right\|_{L^{2}\left(\mathbb{T}^{3}\right)}^{2} T^{\frac{1}{2}}+\left\|\alpha_{k}^{i} \sqrt{1-e^{-2|k|^{2} T}}\right\|_{l_{k, i}^{2}}^{2}}\right),
\end{gathered}
$$

where $\lambda>0, C>0$ and $c>0$ are independent of $T$, and

$$
F_{\lambda, T, u_{0}}=\left\{\omega \in \Omega:\left\|\bar{u}^{\omega}\right\|_{L^{4}\left([0, T] ; L^{6}\left(\mathbb{T}^{3}\right)\right)}+\left\|\nabla \bar{u}^{\omega}\right\|_{L^{2}\left([0, T] ; L^{3}\left(\mathbb{T}^{3}\right)\right)} \geq \lambda\right\} .
$$

Proof. Using a similar argument as that in the proof of (3.1)-(3.2), we can easily obtain (3.4)-(3.5).

Then, using the Bienaymé-Tchebichev inequality, we obtain that there exists $\alpha>0$ such that for every $p \geq 6$ and every $u_{0} \in L^{2}\left(\mathbb{T}^{3}\right)$,

$$
P\left(F_{\lambda, T, u_{0}}\right) \leq \lambda^{-p}\left(\alpha \sqrt{p} G\left(u_{0}\right)\right)^{p},
$$

where $G\left(u_{0}\right)=\left\|u_{0}\right\|_{L^{2}\left(\mathbb{T}^{3}\right)} T^{\frac{1}{4}}+\left\|\alpha_{k}^{i} \sqrt{1-e^{-2|k|^{2} T}}\right\|_{l_{k, i}^{2}}$. If $\lambda$ satisfies

$$
\frac{\lambda}{G\left(u_{0}\right)} \leq 6 \alpha e,
$$

then

$$
C e^{-\left(\frac{c \lambda}{G\left(u_{0}\right)}\right)^{2}} \geq C e^{-(6 c \alpha e)^{2}},
$$

and we can find some $C>0$ and $c>0$ such that

$$
C e^{-(6 c \alpha e)^{2}} \geq 1 \geq P\left(E_{\lambda, u_{0}}\right) \text {. }
$$

Thus inequality (3.6) holds if $\lambda$ satisfies (3.8). If (3.8) does not hold, we set

$$
p:=\left(\frac{\lambda}{\alpha e G\left(u_{0}\right)}\right)^{2} \geq 36
$$


and from (3.7), we obtain

$$
P\left(F_{\lambda, T, u_{0}}\right) \leq\left(\frac{\alpha G\left(u_{0}\right)}{\lambda} \cdot \frac{\lambda}{\alpha e G\left(u_{0}\right)}\right)^{\left(\frac{\lambda}{\alpha e G\left(u_{0}\right)}\right)^{2}}=e^{-\left(\frac{\lambda}{\alpha e G\left(u_{0}\right)}\right)^{2}} .
$$

The following lemma is a classical result of the operator $e^{t \Delta}$ in the three dimensional case.

Lemma 3.3. For any $\alpha$ and $\beta$ satisfying $\frac{2}{\alpha}+\frac{3}{\beta}=3$ and $\beta \in\left(\frac{3}{2}, 2\right]$, we have

$$
\left\|\int_{0}^{t} e^{(t-s) \Delta} f(s, x) d s\right\|_{L^{\infty}\left(0, T ; H^{\frac{1}{2}}\left(\mathbb{T}^{3}\right)\right) \cap L^{2}\left(0, T ; H^{\frac{3}{2}}\left(\mathbb{T}^{3}\right)\right)} \leq C\|f\|_{L^{\alpha}\left(0, T ; L^{\beta}\left(\mathbb{T}^{3}\right)\right)},
$$

where the constant $C$ is independent of $T$.

Proof of Theorem 1.4. If we wish to solve (1.1) with the initial data $u_{0}^{\omega}$ by writing $u=\bar{u}^{\omega}+v$, we get that $v$ solves

$$
\left\{\begin{array}{l}
v_{t}+v \cdot \nabla v-\Delta v+\nabla \Pi=-v \cdot \nabla \bar{u}^{\omega}-\bar{u}^{\omega} \cdot \nabla v-\bar{u}^{\omega} \cdot \nabla \bar{u}^{\omega} \\
\operatorname{div} v=0, x \in \mathbb{T}^{3}, t>0 \\
\left.v\right|_{t=0}=0 .
\end{array}\right.
$$

Write this equation as

$$
v(t, \cdot)=-\int_{0}^{t} e^{(t-s) \Delta} \mathbb{P}\left(v \cdot \nabla v+v \cdot \nabla \bar{u}^{\omega}+\bar{u}^{\omega} \cdot \nabla v+\bar{u}^{\omega} \cdot \nabla \bar{u}^{\omega}\right) d s,
$$

where $\mathbb{P}=\operatorname{Id}+\nabla(-\Delta)^{-1}$ div is the Leray-Hopf projector. Define the map

$$
K^{\omega}: v \mapsto-\int_{0}^{t} e^{(t-s) \Delta} \mathbb{P}\left(v \cdot \nabla v+v \cdot \nabla \bar{u}^{\omega}+\bar{u}^{\omega} \cdot \nabla v+\bar{u}^{\omega} \cdot \nabla \bar{u}^{\omega}\right) d s .
$$

Proposition 3.4. There exists $C>0$ such that for every $T \in(0,1]$, every $u_{0} \in$ $L^{2}\left(\mathbb{T}^{3}\right)$ and every $\lambda>0$, for $\omega \in E_{\lambda, T, u_{0}}^{c}$, the map $K^{\omega}$ satisfies

$$
\begin{gathered}
\left\|K^{\omega}(v)\right\|_{X_{T}^{\frac{1}{2}}} \leq C\left(\lambda^{2}+\|v\|_{X_{T}^{\frac{1}{2}}}^{2}\right), \\
\left\|K^{\omega}\left(v_{1}\right)-K^{\omega}\left(v_{2}\right)\right\|_{X_{T}^{\frac{1}{2}}} \leq C\left\|v_{1}-v_{2}\right\|_{X_{T}^{\frac{1}{2}}}\left(\lambda+\left\|v_{1}\right\|_{X_{T}^{\frac{1}{2}}}+\left\|v_{2}\right\|_{X_{T}^{\frac{1}{2}}}\right),
\end{gathered}
$$

where

$$
\|f\|_{X_{T}^{\frac{1}{2}}}=\|f\|_{L^{\infty}\left([0, T] ; H^{\left.\frac{1}{2}\left(\mathbb{T}^{3}\right)\right)}\right.}+\|\nabla f\|_{L^{2}\left([0, T] ; H^{\left.\frac{1}{2}\left(\mathbb{T}^{3}\right)\right)}\right.} .
$$

Proof. For $\omega \in E_{\lambda, T, u_{0}}^{c}, T \in(0,1]$, we have

$$
\left\|\bar{u}^{\omega}\right\|_{L^{4}\left([0, T] ; L^{6}\left(\mathbb{T}^{3}\right)\right)}+\left\|\nabla \bar{u}^{\omega}\right\|_{L^{2}\left([0, T] ; L^{3}\left(\mathbb{T}^{3}\right)\right)}<\lambda .
$$

Then, we obtain

$$
\begin{gathered}
\|v \cdot \nabla v\|_{L^{\frac{4}{3}\left([0, T] ; L^{2}\right)}} \leq C\|v\|_{L^{4}\left([0, T] ; L^{6}\right)}\|\nabla v\|_{L^{2}\left([0, T] ; L^{3}\right)} \leq C\|v\|_{X_{T}^{\frac{1}{2}}}^{2}, \\
\left\|v \cdot \nabla \bar{u}^{\omega}\right\|_{L^{\frac{4}{3}\left([0, T] ; L^{2}\right)}} \leq C\|v\|_{L^{4}\left([0, T] ; L^{6}\right)}\left\|\nabla \bar{u}^{\omega}\right\|_{L^{2}\left([0, T] ; L^{3}\right)} \leq C \lambda\|v\|_{X_{T}^{\frac{1}{2}}}, \\
\left\|\bar{u}^{\omega} \cdot \nabla v\right\|_{L^{\frac{4}{3}\left([0, T] ; L^{2}\right)}} \leq C\left\|\bar{u}^{\omega}\right\|_{L^{4}\left([0, T] ; L^{6}\right)}\|\nabla v\|_{L^{2}\left([0, T] ; L^{3}\right)} \leq C \lambda\|v\|_{X_{T}^{\frac{1}{2}}}, \\
\left\|\bar{u}^{\omega} \cdot \nabla \bar{u}^{\omega}\right\|_{L^{\frac{4}{3}\left([0, T] ; L^{2}\right)}} \leq C\left\|\bar{u}^{\omega}\right\|_{L^{4}\left([0, T] ; L^{6}\right)}\left\|\nabla \bar{u}^{\omega}\right\|_{L^{2}\left([0, T] ; L^{3}\right)} \leq C \lambda^{2} .
\end{gathered}
$$


Then, using Lemma 3.3, we can easily obtain that

$$
\begin{aligned}
& \left\|K^{\omega}(v)\right\|_{X_{T}^{\frac{1}{2}}} \\
\leq & C\left(\|v \cdot \nabla v\|_{L_{T}^{\frac{4}{3}} L_{x}^{2}}+\left\|v \cdot \nabla \bar{u}^{\omega}\right\|_{L_{T}^{\frac{4}{3}} L_{x}^{2}}+\left\|\bar{u}^{\omega} \cdot \nabla v\right\|_{L_{T}^{\frac{4}{3}} L_{x}^{2}}+\left\|\bar{u}^{\omega} \cdot \nabla \bar{u}^{\omega}\right\|_{L_{T}^{\frac{4}{3}} L_{x}^{2}}\right) \\
\leq & C\left(\lambda^{2}+\|v\|_{X_{T}^{\frac{1}{2}}}^{2}\right) .
\end{aligned}
$$

Similarly, we have

$$
\begin{aligned}
& \left\|K^{\omega}\left(v_{1}\right)-K^{\omega}\left(v_{2}\right)\right\|_{X_{T}^{\frac{1}{2}}} \\
\leq & C\left(\left\|\left(v_{1}-v_{2}\right) \cdot \nabla v_{1}\right\|_{L_{T}^{\frac{4}{3}} L_{x}^{2}}+\left\|v_{2} \cdot \nabla\left(v_{1}-v_{2}\right)\right\|_{L_{T}^{\frac{4}{3}} L_{x}^{2}}\right. \\
& \left.+\left\|\left(v_{1}-v_{2}\right) \cdot \nabla \bar{u}^{\omega}\right\|_{L_{T}^{\frac{4}{3}} L^{2}}+\left\|\bar{u}^{\omega} \cdot \nabla\left(v_{1}-v_{2}\right)\right\|_{L_{T}^{\frac{4}{3}} L_{x}^{2}}\right) \\
\leq & C\left\|v_{1}-v_{2}\right\|_{X_{T}^{\frac{1}{2}}}\left(\lambda+\left\|v_{1}\right\|_{X_{T}^{\frac{1}{2}}}+\left\|v_{2}\right\|_{\left.X_{T}^{\frac{1}{2}}\right) .}\right.
\end{aligned}
$$

Let us now complete the proof of Theorem 1.4. Define

$$
\Sigma=\bigcup_{n=1}^{\infty} E_{\lambda_{1}, \frac{1}{n}, u_{0}}^{c}
$$

where $\lambda_{1}$ is a positive constant satisfying

$$
C \lambda_{1}^{2}+C\left(2 C \lambda_{1}^{2}\right)^{2} \leq 2 C \lambda_{1}^{2} \text { and } C \lambda_{1}+2 C\left(2 C \lambda_{1}^{2}\right) \leq \frac{1}{2} .
$$

From (3.3) we have

$$
P\left(E_{\lambda_{1}, \frac{1}{n}, u_{0}}^{c}\right) \geq 1-C \lambda_{1}^{-6}\left(\left\|u_{0}\right\|_{L^{2}\left(\mathbb{T}^{3}\right)} n^{-\frac{1}{4}}+\left\|\alpha_{k}^{i} \sqrt{1-e^{-2|k|^{2} / n}}\right\|_{l_{k, i}^{2}}\right)^{6} \rightarrow 1, \text { as } n \rightarrow \infty .
$$

Thus, we have

$$
P(\Sigma)=1 .
$$

For any $\omega \in \Sigma$, there exists $n$ such that $\omega \in E_{\lambda_{1}, \frac{1}{n}, u_{0}}^{c}$ and

$$
\left\|\bar{u}^{\omega}\right\|_{L^{4}\left(\left[0, \frac{1}{n}\right] ; L^{6}\left(\mathbb{T}^{3}\right)\right)}+\left\|\nabla \bar{u}^{\omega}\right\|_{L^{2}\left(\left[0, \frac{1}{n}\right] ; L^{3}\left(\mathbb{T}^{3}\right)\right)}<\lambda_{1} .
$$

From Proposition 3.4 we have that the map $K^{\omega}$ is a contraction on the ball of radius $2 C \lambda_{1}^{2}$ of $X_{\frac{1}{n}}^{\frac{1}{2}}$. Thus, for almost all $\omega \in \Omega$, there exist $T_{\omega}>0$ and a unique solution $v \in L^{\infty}\left(\left[0, T_{\omega}\right] ; H^{\frac{1}{2}}\left(\mathbb{T}^{3}\right)\right) \cap L^{2}\left(\left[0, T_{\omega}\right] ; H^{\frac{3}{2}}\left(\mathbb{T}^{3}\right)\right)$ to (3.9). Since it is easy to obtain that $v \in C\left(\left[0, T_{\omega}\right] ; H^{\frac{1}{2}}\left(\mathbb{T}^{3}\right)\right)$, we obtain the first part in Theorem 1.4.

When $\left\{h_{k}^{i}\right\}_{k \in \mathbb{Z}_{*}^{3}, i=1,2}$ satisfies (1.9), let

$$
\Omega_{T}=E_{\lambda_{1}, T, u_{0}}^{c} .
$$

From (3.3) and a similar argument as above, we have (1.10) and the fact that there exists a unique solution $u^{\omega} \in X_{\omega}$ of (1.1) with the initial data $u_{0}^{\omega}$ in the time interval $[0, T]$.

When $h_{k}^{i}$ are standard real Gaussian or Bernoulli variables, let

$$
\Omega_{T}=F_{\lambda_{1}, T, u_{0}}^{c} .
$$

From (3.6) and a similar argument as above, we have (1.11) and the fact that there exists a unique solution $u^{\omega} \in X_{\omega}$ of (1.1) with the initial data $u_{0}^{\omega}$ in the time interval $[0, T]$. If there is a solution $u_{2} \in C\left([0, T] ; L^{2}\left(\mathbb{T}^{3}\right)\right) \cap L^{2}\left([0, T] ; H^{1}\left(\mathbb{T}^{3}\right)\right)$ of 
(1.1) with the initial data $u_{0}^{\omega}$, using the energy method, one can easily obtain that $u^{\omega}=u_{2}$ a.e. and finish the proof of Theorem 1.4.

\section{ACKNOWLEDGMENT}

The authors would like to thank the referee for helpful suggestions about the manuscript and for telling them about the Khinchin inequality.

\section{REFERENCES}

1. H. Amann, On the strong solvability of the Navier-Stokes equations, J. Math. Fluid Mech., 2 (2000), 16-98. MR 1755865 (2002b:76028)

2. A. Ayache, N. Tzvetkov, $L^{p}$ properties of Gaussian random series, Trans. Amer. Math. Soc., 360 (2008), 4425-4439. MR2395179 (2009e:30006)

3. N. Burq, N. Tzvetkov, Random data Cauchy theory for supercritical wave equations I. Local theory, Invent. Math., 173 (2008), 449-475. MR2425133 (2009k:58057)

4. M. Cannone, Y. Meyer, F. Planchon, Solutions autosimilaires des équations de Navier-Stokes, Séminaire "Équations aux Dérivées Partielles" de l'École Polytechnique, Exposé VIII, 19931994. MR.1300903 (95k:35157)

5. R. Farwig, H. Sohr, Optimal initial value conditions for the existence of local strong solutions of the Navier-Stokes equations, Math. Ann., 345 (2009), 631-642. MR2534111

6. H. Fujita, T. Kato, On the Navier-Stokes initial value problem, Arch. Ration. Mech. Anal., 16 (1964), 269-315. MR0166499(29:3774)

7. Y. Giga, Solutions for semilinear parabolic equations in $L^{p}$ and regularity of weak solutions of the Navier-Stokes system, J. Differ. Equ., 61 (1986), 186-212. MR833416 (87h:35157)

8. E. Hopf, Über die Anfangswertaufgabe für die hydrodynamischen Grundgleichungen, Math. Nachr., 4 (1950-51), 213-231. MR0050423 (14:327b)

9. T. Kato, Strong $L^{p}$-solutions of the Navier-Stokes equation in $\mathbb{R}^{m}$, with applications to weak solutions, Math. Z., 187 (1984), 471-480. MR760047(86b:35171)

10. A.Y. Khinchin, Ueber dyadische Brüche, Math. Z., 18 (1923), 109-116. MR.1544623

11. A.A. Kiselev, O.A. Ladyzenskaya, On the existence of uniqueness of solutions of the nonstationary problems for flows of non-compressible fluids, Amer. Math. Soc. Transl. Ser. 2., 24 (1963), 79-106.

12. H. Koch, D. Tataru, Well-posedness for the Navier-Stokes equations, Adv. Math., 157 (2001), 22-35. MR $1808843(2001 \mathrm{~m}: 35257)$

13. J. Leray, Sur le mouvement d'un liquide visqueux emplissant l'espace, Acta Math., 63 (1934), 193-248. MR 1555394

14. R.E.A.C. Paley, A. Zygmund, On some series of functions (1) (2) (3), Proc. Cambridge Philos. Soc., 26 (1930), 337-357, 458-474, 28 (1932), 190-205.

Department of Mathematics, Zhejiang University, Hangzhou 310027, People's RepubLIC OF CHINA

E-mail address: zhangting79@zju.edu.cn

Department of Mathematics, Zhejiang University, Hangzhou 310027, People's RepubLIC OF CHINA

E-mail address: dyf@zju.edu.cn 